\title{
Research and Design of Internet of Things Information Monitoring System Based on Fiber Optic Sensing
}

\author{
LU Jun ${ }^{1,}$, , WU Zhengnan ${ }^{2}$ \\ ${ }^{1,2}$ School of Electronics and Information Engineering, Wuhan Donghu University, HuBei Wuhan, \\ 430212, China \\ a bdbpx@163.com
}

Keywords: Internet of Things; fiber sensing; distributed feedback fiber lasers (DFB-FL); phase generated carrier(PGC); virtual instruments

\begin{abstract}
The Internet of things is network connected things. Internet of things is generally divided into three layers, perception layer, transmission network layer and application layer. Sensing end of the sensing layer is the electric sensor mostly used currently. But with the rapid development of optical fiber communication technology in recent years, fiber optic sensor gradually entered people's field of vision and applied in the Internet of things. Optical fiber sensor use light to carry information by tacking optical fiber as transmission medium, which therefore of the advantages of both optical measurement and optical fiber transmission.
\end{abstract}

\section{Related concepts about Internet of things}

\section{Definition of Internet of things}

Internet of things is a kind of intelligent network that access any object to the existing communication network, in order to realize the real-time monitoring and management through all kinds of information sensing device through wired and wireless access. Internet of things can be seen as a product of sensor network and the Internet, but not the simple physical fusion, it is deeper integration and development. It is widely acknowledged that the Internet of things is the world's information industry after computer technology, Internet technology of the third wave. Internet makes people can achieve fast and convenient communication between each other in different parts. Era of Internet of things can further realize between people and things, and even content and content of information exchange.

\section{Origination of the Internet of things}

In 1995, Bill Gates wrote the idea of the Internet of things in his book The Road to the Future, in which the idea of something connected was mentioned. But limited to relevant technology needed for the Internet of things is not yet mature, his this idea at the time did not cause the attention of people.

In 1999, the Massachusetts institute of technology (MIT) Auto - ID Labs put forward that the object with the radio frequency identification (RFID) device, which is then connected to the Internet, can realize the intelligent management of object. In the same year, mobile computing and networking international conference held in the United States, the concept of Internet of things for the first time appeared in the international conference, but the main support technology is a radio frequency identification technology and the wireless sensor network technology. From then on, the Internet of things officially entered people's vision.

\section{Architecture and related technologies of the Internet of things}

There are different kinds of classification methods about the architecture of Internet of things; three layer models are used in this paper: perception, transmission network layer and application layer.

Sense layer: You can also call it a sensor network layer, which is mainly composed of various kinds of sensor devices and microprocessors to perceive and acquisition in the environment of various physical quantities or items identified parameters. [1] It involves the technology of sensor technology, radio frequency identification technology (RFID), wireless sensor network (wireless sensor network, WSN), cable sensor network, etc. 
Transmission network layer, transport layer is mainly for processing and transmission of the sensing information on perception layer. In particular it can be subdivided into bearing layer and access layer.

Application layer: it is for judgment, processing and usage of data collected from the transport layer, including all kinds of application server (database server, web server, etc.), cloud computing platform, and the client. It involves in database related technology, web technology and so on.

\section{Optical fiber sensing technology and its application}

The basic structure of the optical fiber sensing system

Optical fiber sensing system generally consists of light source, optical fiber, optical fiber sensors, photoelectric detection equipment, signal processing circuit, etc., as shown in the figure below.

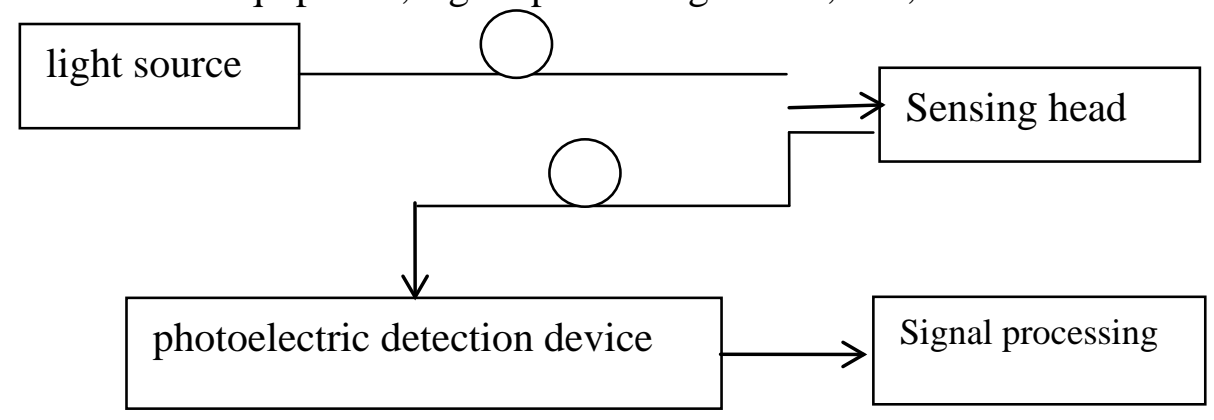

Figure 1 Typical optical fiber sensing system

\section{Active and passive sensing}

With the high-speed development of economy, people's demand of multi-classification is higher and higher. The application of the sensor is increasingly widely used, which can be detected by measuring converted to facilitate processing of signal such as electric signals and optical signals. Broadly speaking, the sensor is the device that changes the electric energy like physical or chemical energy into electrical signal. According to the different ways of energy conversion, sensor can be divided into active and passive sensors.

Active sensor can directly convert one form of energy into another form of energy without requiring additional incentives, so active sensors are also known as energy conversion sensor or transducer. As shown in figure 2:

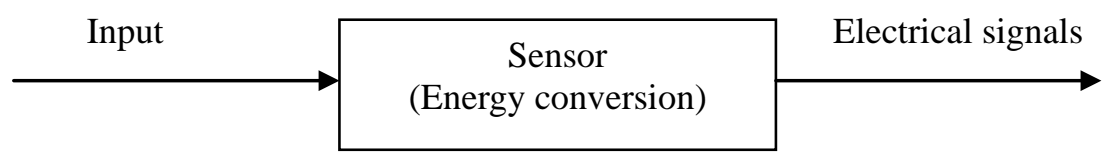

Figure 2 The basic principle of active sensors

Passive sensors can only directly conduct conversion of energy after getting a excitation energy. Input of passive sensor input is commonly some characteristics or properties of an object. Through the passive sensors, we can turn this is not a quantitative characteristics into electricity energy measurement purposes. [2]As shown in figure 3:

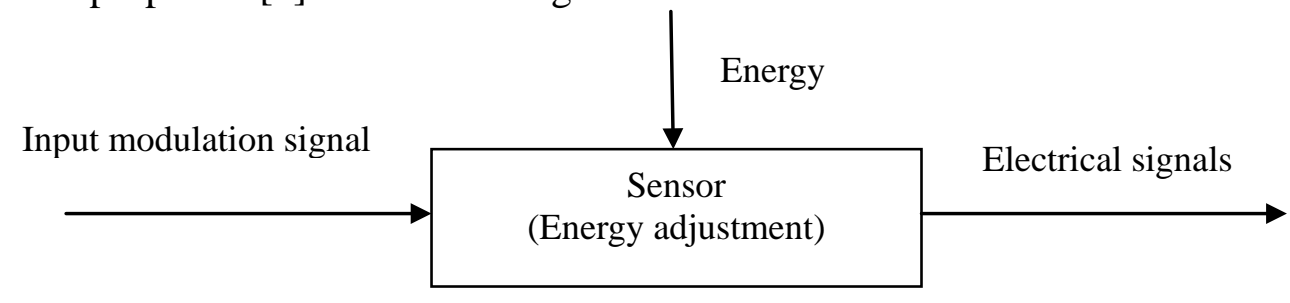

Figure3 The basic principle of passive sensors 


\section{Distributed-feedback fiber laser}

\section{1) Introduction about distributed-feedback fiber laser}

Analysis of the working principle and characteristics of distributed laser in abroad began in 1972, Bell LABS in the United States. They successfully used the fuel production grating on glass substrate. With the development of the time, people can reduce the threshold effect according to the embedded in distributed feedback semiconductor laser grating through introduction of the theory of discontinuous changes on the laser is applied to implement the single-mode laser work.

\section{2) Working principle of distributed-feedback fiber laser as the transducer}

In recent years DFB - FL fiber laser was used as a preferred source for high sensitivity and precision of measurement because of its long transmission distance, high SNR, line width, tuning range width, and the advantages of stable single mode output. [3]We can take advantage of the DFB - FL for dynamic strain of the weak signal detection, which the general broadband light source incapable of.

When environment such as temperature, pressure, sound waves is changed, then the DFB laser wavelength will be impacted. When only a voice signal on the DFB - FL laser, DFB - FL response to external strain or temperature and all other same as Bragg grating. Only considering axial strain, the Bragg grating wavelength change as follows:

$$
\frac{\Delta \lambda_{B}}{\lambda_{B}}=\varepsilon_{z}-\frac{n_{e f f}^{2}}{2}\left[P_{12} \varepsilon_{z}+\left(P_{11}+P_{12}\right) \varepsilon_{r}\right]
$$

Relations between DFB - FL output wavelength type strain and the outside world also meet type above. $n_{\text {eff }}$ is for the effective refractive index; $P_{11}$ and $P_{12}$ as elastic-optic coefficient of the fiber. Of quartz optical fiber, $n_{\text {eff }}$ is 1.456, $P_{11}$ and $P_{12}$ is 0.121 and 0.265 . When external noise signal, DFB - FL fiber radial and transverse strain can be generated to $\varepsilon_{z}$ and $\varepsilon_{r}$ respectively.

$$
\varepsilon_{z}=\varepsilon_{r}=\frac{(2 v-1) P}{E}
$$

E is young's modulus; $\quad v$ for Poisson's ratio; $\mathrm{P}$ for acoustic pressure:

$$
\Delta \lambda=\lambda\left[1-\frac{n_{e f f}^{2}}{2}\left(P_{11}+2 \times P_{12}\right)\right] \frac{(2 v-1)}{E} P
$$

\section{Non-equilibrium MZI demodulation system based on carrier phase method}

It is known that output photo current of M-Z interferometer system is proportional to the light intensity, need to get the demodulation and signal phase information related to the outside world but there is the size of the random phase decline phenomenon.

\section{Principle of carrier phase method}

Experiments use carrier phase demodulation method to realize the wavelength demodulation. PGC detecting way make signal under test its sideband by introducing a carrier signal phase, then use a series of signal processing method to handle interferometer output electric signal, split the ac signal (including external signal under test), and the stability of the sensor signal is obtained.

Based on carrier phase method of the $\mathrm{M}-\mathrm{Z}$ interferometer demodulation system, output light intensity of interferometer interference of can be as:

$$
I=A+B \cos \Phi(t)
$$

On the type above, $\mathrm{A}$ and $\mathrm{B}$ is constant, directly proportional to the intensity of the input, and meet $B=\gamma A$. Make $\Phi(t)=\phi(t)+\phi_{r}(t)+\phi_{o}(t)$, then:

$$
I=A+B \cos \left(\phi(t)+\phi_{r}(t)+\phi_{o}(t)\right)
$$

Suppose load on the piezoelectric ceramics (PZT) voltage signal $\varphi(t)=\varphi_{r}(t)+\varphi_{o}(t)$, then piezoelectric effect generated by interference phase difference is:

$I=A+B \cos \left(C \cos \left(\omega_{o} t\right)+\phi(t)\right)$

After Bessel function, the type above can be expressed as: 


$$
\begin{aligned}
I=A+ & B\left\{\left[J_{o}(C)+2 \sum_{n=1}^{\infty}(-1)^{m} J_{2 n}(C) \cos \left(2 n \omega_{o} t\right)\right] \cos (\varphi(t))\right. \\
& \left.-2\left[\sum_{n=0}^{\infty}(-1)^{n} J_{2 n+1}(C) \cos \left((2 n+1) \omega_{o} t\right)\right] \sin (\varphi(t))\right\}
\end{aligned}
$$

\section{Implementation method of carrier phase}

PGC demodulation principle block diagram can be used to realize PGC algorithm. [4]The implementation of the general algorithm are mainly of two ways, the hardware and software ways: hardware implementation is to interference output photo current signal acquisition directly to hardware circuit (such as DSP circuit, analog circuit design), and then realize the algorithm, complete the demodulation, further can finish hardware circuit demodulation signal distance set in the computer, do the storage and release, etc., to meet the requirements of the Internet of things; Software way, was the light of the interfere-meter output signal is converted into electrical signals, after data acquisition card, collecting directly to the computer, the computer software to complete demodulation, storage and release further.

\section{Determination of key parameters signal and sampling rate in the experiment}

\section{1) The parameters selection of the phase carrier}

As for the selection of the magnitude of Carrier phase $C$ value: value of $C$ should ensure that the final demodulation signal are stable output, two points need to be taken into consideration: one is to make the largest output, the second is to make sure that even if the carrier signal disturbed by some small changes and make the $\mathrm{C}$ value would not result in great fluctuations of demodulation of the signal. This request $C$ must satisfied: $\left.J_{1}(C) J_{2}(C)\right|_{C}=\operatorname{Max}$, at the same time $\left.\left[J_{1}(C) J_{2}(C)\right]^{\prime}\right|_{C}=0$. After computing and comparison, while $\mathrm{C}=2.37$ can meet the requirements.

Carrier frequency selection: the frequency of the signal under test in the experiment is $1 \mathrm{kHz}$, carrier frequency $\omega_{o}$ is $10 \mathrm{kHz}$, satisfy the requirement of far away from the side-band signal under test.

\section{2) Sampling frequency}

The realization of the digital must convert analog signal into digital signal, which requires to collect the original signal at a certain frequency. According to the Nyquist sampling theorem, the sampling frequency should be at least 2 times of the original signal frequency to guarantee the signal avoid from distortion. Because the frequency of the carrier is $10 \mathrm{kHz}$, the calculation sample frequency should be at least $20 \mathrm{kHz}$, taking into account other factors such as filter demodulation of some influence, can take to $100 \mathrm{kHz}$.

\section{Summary}

With the development of related technologies, the Internet of things has became the research hot spot, the combination of optical fiber sensing technology and web technology has especially become a new research direction. Based on the three aspects of the Internet of things, namely sensing layer, network layer and application layer, this article put forward a optical fiber sensing system for the application of Internet of things targeting at sensor node. Sensing layer adopt the distributed-feedback fiber laser DFB-FL as sensing head of fiber optic sensor. the article simply analyzes the DFB-FL working principle and the demodulation method of taking DFB-FL as sensing head of fiber optic sensing system. Digital carrier phase demodulation system based on the non-equilibrium $\mathrm{M}-\mathrm{Z}$ interferometer and virtual instrument is designed, and the data delivery platform based on $\mathrm{B} / \mathrm{S}$ architecture is completed.

\section{Acknowledgements}

Hubei Provincial Department of Education Scientific and technological research projects (B2014145). 


\section{References}

[1] Texas Instruments.Code Composer Studio Development Tools v3.3 Getting Started Guide, 2006.

[2] Dandridge A, Tveten A B, Kersey A D, et al. Multiplexing of interferometric sensors using phase carrier techniques[J]. Lightwave Technology, Journal of, 1987,

[3] Cranch G A, Flockhart G M H,Kirkendall C K. Distributed feedback fiber laser strain sensors[J]. Sensors Journal, IEEE,2008, 8(7): 1161-1172.

[4] Davis M A,Kersey A D. Matched-filter interrogation technique for fibre Bragg grating arrays[J]. Electronics letters,1995, 31(10): 822-823. 